\title{
Development of a rotating-coil scanner for superconducting accelerator magnets
}

\author{
Piotr Rogacki ${ }^{1,2}$, Lucio Fiscarelli ${ }^{1}$, Stephan Russenschuck ${ }^{1}$, Kay Hameyer $^{2}$ \\ ${ }^{1}$ CERN, Switzerland \\ ${ }^{2}$ Institute of Electrical Machines (IEM), RWTH Aachen University, Aachen, Germany \\ p.rogacki@cern.ch
}

\begin{abstract}
The superconducting magnets for High-Luminosity Large Hadron Collider (HL-LHC) upgrade project at CERN require magnetic measurements with high precision in field angle and multipole field errors. The paper describes the design, development, and metrological characterization of a new, rotating-coil magnetometer and a transport system to scan the magnetic field along the axis of the accelerator magnet.
\end{abstract}

Keywords: Magnets, Accelerators, PCB, Magnetometer, 3D Printing

\section{Introduction}

The High-Luminosity upgrade of the Large Hadron Collider (HL-LHC) [1] requires $\mathrm{Nb}_{3} \mathrm{Sn}$ superconducting magnets with strict requirements for their field quality. Therefore, the magnets must be measured with highest possible precision of up to 1-ppm levels, both locally and averaged over their entire length of up to ten meters. To acquire all required quantities now, the superconducting quadrupole magnets have to be measured using both rotating-coil magnetometers [5] and stretched-wire systems [7]. It is therefore advantageous to develop a new magnetometer for the upcoming measurement campaign that allows us to measure all required quantities simultaneously.

\section{Harmonic fields and rotating-coil magne- tometers}

A 2D magnetic field in a domain that is free of current and magnetized material (such as the bore of accelerator magnets) can be described as a complex valued harmonic field expansion [9]:

$$
B_{y}+\mathrm{i} B_{x}=\sum_{n=1}^{\infty}\left(B_{n}+\mathrm{i} A_{n}\right)\left(\frac{x+\mathrm{i} y}{r_{0}}\right)^{n-1},
$$

where $r_{0}$ is the reference radius, and $B_{n}$ and $A_{n}$ are called the normal and skew harmonic coefficients, or in short, field multipoles. In practice, the $n$-th coefficient corresponds to a field generated by a magnet with $n^{1}$ pole pairs, for example, for a quadrupole magnet $n=2$.

\footnotetext{
1 This convention, established for the accelerator magnets, differs from the usual electrical machines naming convention in using $n$ instead of $p$ for the pole pair number.
}

In the case of accelerator magnets, the field along the magnet length can be integrated and shown to satisfy the two-dimensional Laplace equation. The same applies to the field integrated along the length of an induction coil, with the constraint that the ends of the coil are in the domain that is free of axial field components. Otherwise, the coil ends will pick up the field in the axial $(z)$ direction, in which case the measurement results do not correspond to the 2D mathematical model but must be treated with the theory of pseudo-multipoles [2].

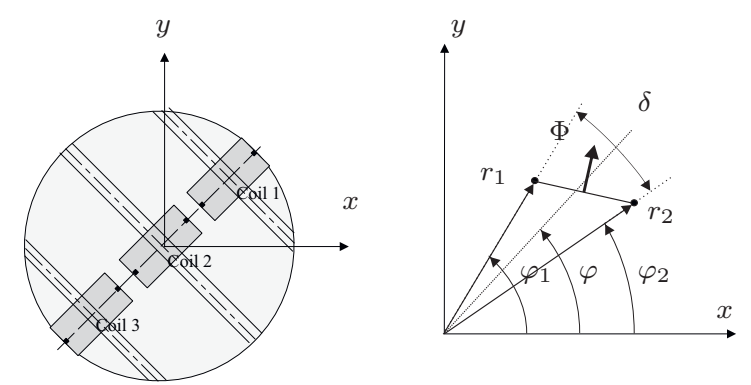

Fig. 1: Left: Radial (sagital) coil array. Right: Naming convention for angles and radii of a single wire loop.

The rotating-coil measurement principle is especially suited to characterize the fields described as in Eq. 1. This is due to the fast and robust analysis of the output signals.

According to Faraday's law:

$$
U=-\frac{\mathrm{d} \Phi_{B}}{\mathrm{~d} t},
$$

using the principal coil arrangement in the shaft and the geometric relations shown in Fig. 1, we 
can calculate the magnetic flux intercepted by the induction coil.

Knowing that [9]

$$
\begin{aligned}
\int_{z_{1}}^{z_{2}} \boldsymbol{B}(\boldsymbol{z}) \mathrm{d} \boldsymbol{z}= & \int_{z_{1}}^{z_{2}}\left(B_{y} \mathrm{~d} x-B_{x} \mathrm{~d} y\right) \\
& +\mathrm{i} \int_{z_{1}}^{z_{2}}\left(B_{y} \mathrm{~d} y+B_{x} \mathrm{~d} x\right),
\end{aligned}
$$

the flux intercepted by the induction coil can be expressed as

$$
\begin{aligned}
\Phi & =N \ell \operatorname{Re}\left\{\int_{z_{1}}^{z_{2}} \boldsymbol{B}(\boldsymbol{z}) \mathrm{d} \boldsymbol{z}\right\} \\
& =N \ell \operatorname{Re}\left\{\int_{z_{1}}^{z_{2}} \sum_{n=1}^{\infty}\left(B_{n}+\mathrm{i} A_{n}\right)\left(\frac{\boldsymbol{z}}{r_{0}}\right)^{n-1} \mathrm{~d} \boldsymbol{z}\right\} \\
& =\operatorname{Re}\left\{\sum_{n=1}^{\infty} \frac{N \ell}{n r_{0}^{n-1}}\left(B_{n}+\mathrm{i} A_{n}\right)\left(z_{2}^{n}-z_{1}^{n}\right)\right\} \\
& =\operatorname{Re}\left\{\sum_{n=1}^{\infty} C_{\boldsymbol{n}} S_{\boldsymbol{n}} e^{\mathrm{i} n \varphi}\right\}
\end{aligned}
$$

where $C_{n}:=B_{n}+\mathrm{i} A_{n}$ (at the reference radius $r_{0}$ ) and the $S_{n}$ are complex valued sensitivity factors given by

$$
\begin{aligned}
\boldsymbol{S}_{\boldsymbol{n}}\left(r_{0}\right) & =S_{n}^{\mathrm{rad}}-\mathrm{i} S_{n}^{\tan } \\
& =\frac{N \ell}{n r_{0}^{n-1}}\left(\boldsymbol{z}_{2}^{n}-\boldsymbol{z}_{1}^{n}\right) \\
& =\frac{N \ell}{n r_{0}^{n-1}}\left(r_{2}^{n} e^{\mathrm{i} n\left(\varphi_{2}-\varphi\right)}-r_{1}^{n} e^{\mathrm{i} n\left(\varphi_{1}-\varphi\right)}\right) .
\end{aligned}
$$

Radial $^{2}$ coils, as sketched in Fig. 1 (left), intercept the azimuthal component of the magnetic flux density. For $\varphi_{1}=\varphi_{2}=\varphi$, inner radius $r_{1}$ and outer radius $r_{2}$ the $S_{n}^{\tan }$ are zero and we obtain:

$$
\boldsymbol{S}_{\boldsymbol{n}}=S_{n}^{\mathrm{rad}}=\frac{N \ell}{n r_{0}^{n-1}}\left(r_{2}^{n}-r_{1}^{n}\right) .
$$

The $S_{n}^{\text {rad }}$ are calculated using the geometry of the coils and their arrangement in the probe. In the most straightforward case the field multipoles can then be calculated using

$$
B_{n}+\mathrm{i} A_{n}=\frac{\psi_{\boldsymbol{n}}}{\boldsymbol{K}_{\boldsymbol{n}}},
$$

where $\boldsymbol{K}_{\boldsymbol{n}}=\boldsymbol{S}_{\boldsymbol{n}} r_{0}^{n-1}$ are the sensitivity factors defined to be independent of the reference radius and $\psi_{n}$ are complex coefficients of a Fourier transform of the acquired integrated voltage.

The harmonic content of the field is used as one of the quality measures. Generally, a field of an acceptable quality is defined to have at most a few hundred ppm of multipole coefficients.

In realistic measurement, affected by mechanical imperfections and signal noise, some additional steps are required to achieve the specified accuracy. First of all, for the integration of voltage it is necessary to keep exact track of the timing. This is complicated due to the instabilities in shaft rotation caused by the motor and bearings. They introduce vibrations, which can influence the measured field harmonics. The established solution to that problem is the use of digital integrators triggered by an angular encoder coupled with the shaft. The voltage between two triggers is integrated, yielding a measure of the flux linked between the two positions. Mathematically this corresponds to a re-parametrization of the signal with respect to the rotation angle instead of time. Multiple coils in the probe can be connected together in an appropriate configuration to provide compensation of the main field components [10]; in our case the quadrupole and dipole field components. This allows to amplify the compensated signal, thus increasing the SNR for the higherorder field harmonics. Moreover, compensation may reduce the impact of mechanical vibrations and displacements, if all connected coils move together.

Finally, to correct the misalignment of the measurement coils with the magnetic axis, a so called feed-down correction is applied [9]. In the case of a centered quadrupole measurement, the dipole component should vanish, except if the coil is decentered with respect to the magnetic axis. This misalignment can be calculated and corrected by translating the reference frame of the results to where the dipole harmonic is minimized. This yields the centered field harmonic coefficients as well as the position of the magnetic field center in the local sensor coordinates.

\section{Requirements}

The requirements for the measurement system are based on the design of the HL-LHC magnets and the accuracy specifications laid out in table 1.

Tab. 1: The target accuracy for LHC insertion region quadrupole measurements [1].

\begin{tabular}{|l|c|c|}
\hline Parameter & Unit & Accuracy \\
\hline Main field & {$[\mathrm{ppm}]$} & 100 \\
Main field direction & {$[\mathrm{mrad}]$} & 0.1 \\
Harmonics & {$[\mathrm{ppm}]$} & 1 \\
Magnetic center & {$[\mathrm{mm}]$} & 0.15 \\
\hline
\end{tabular}

The measurement system must be able to measure different quantities with highest achievable accuracy. All those quantities must be provided

${ }^{2}$ Or better sagital. 
both locally and integrated along the entire length of the magnet (up to $10 \mathrm{~m}$ ). They are important as they allow to intercept manufacturing errors at an earliest state in the production.

The two types of systems commonly used for magnetic measurements of long accelerator magnets are rotating-coil magnetometers and stretched-wire systems. A summary of the currently achievable measurement accuracy for those systems is given in table 2 .

Tab. 2: Existing systems accuracy [3, 6, 7, 8]

\begin{tabular}{|l|c|l|l|l|}
\hline Parameter & Accuracy & Units & Type & System \\
\hline Main field & $\begin{array}{c}100 \\
1000\end{array}$ & {$[\mathrm{ppm}]$} & $\begin{array}{l}\text { Integral } \\
\text { Local }\end{array}$ & $\begin{array}{l}\text { Stretched wire } \\
\text { Rotating coils }\end{array}$ \\
\hline $\begin{array}{l}\text { Magnetic } \\
\text { center }\end{array}$ & 0.05 & {$[\mathrm{~mm}]$} & $\begin{array}{l}\text { Integral } \\
\text { Local }\end{array}$ & $\begin{array}{l}\text { Stretched wire } \\
\text { Rotating coils }\end{array}$ \\
\hline $\begin{array}{l}\text { Main field } \\
\text { direction }\end{array}$ & 0.1 & {$[\mathrm{mrad}]$} & $\begin{array}{l}\text { Integral } \\
\text { Local }\end{array}$ & $\begin{array}{l}\text { Stretched wire } \\
\text { Rotating coils }\end{array}$ \\
\hline Harmonics & 1 & {$[\mathrm{ppm}]$} & $\begin{array}{l}\text { Integral } \\
\text { Local }\end{array}$ & $\begin{array}{l}\text { Rotating coils } \\
\text { Rotating coils }\end{array}$ \\
\hline
\end{tabular}

The accuracy for the integral field-center and field-direction measurements has been obtained with a Single-Stretched Wire system as used for the LHC main quadrupoles [7]. These magnets posed similar challenges as the ones to be measured in the incoming HL-LHC upgrade project. The expected accuracy for the field harmonics measurement are based on the probes developed by Fermi National Accelerator Laboratory (FNAL) and Lawrence Berkeley National Laboratory (LBNL) for the LHC Accelerator Research Program (LARP) as described in [6]. These probes are designed for measuring a highgradient quadrupole magnet and are based on a similar principle, with the use of induction coils produced in PCB technology. The accuracy of the other parameters retrieved by rotating-coil systems are based on the performance of the legacy QIMM and DIMM systems, used for LHC dipole and quadrupole measurements at ambient temperatures [8].

It can be observed that even though for most parameters a system exists that allows a measurement with the required accuracy, it is necessary to use at least two or three complementary systems to measure and derive all required data. Given the considerable time and effort for each measurement, a new system had to be developed, able to measure all aforementioned quantities simultaneously.

Additionally, as the new magnets will have multiple different lengths and apertures, the system needs to be easily adaptable. The size and radius of the induction coils have an influence on the amplitude of the acquired signal and thus on the signal-to-noise ratio (SNR). This is especially important for magnets such as the quadrupoles, sextupoles and higher orders.

\section{The prototype magnetometer}

Taking into account all the requirements as listed above, the necessity for the local and integral measurements of long magnets, and the required accuracy of the measurements, the best approach was to design a new rotating-coil scanner, able to provide all values locally, and after scanning the entire magnet bore, providing the integral values as well.

By comparing the specification with the designs of existing rotating-coil scanners, like the QIMM [8] or the ferret [6], we derived the main design concepts:

- Construction adaptable to magnet apertures ranging from $90 \mathrm{~mm}$ to $150 \mathrm{~mm}$ and above,

- 3D printing for complicated structural parts,

- built in encoder and level meter,

- PCB based induction coils,

- open end for mounting retro-reflectors for the laser tracker,

- and transport in the magnet bore with extension rods.

The placing of the encoder and the level meter close to the induction coils is necessary to precisely track their angular position, that in turn enables the accurate measurement of the angle of the magnetic field. In order to relate the magnetic-axis measurement (given in the probe coordinates) to the magnet reference, one end of the scanner must be open to leave free lineof-sight for the laser tracker. During the rotation, the tracker follows the retro-reflectors mounted on the shaft. By fitting a circle to the measured points, it is possible to find the mechanical rotation axis and indirectly the axis of the magnetic field. The longitudinal positioning is realized by the use of extension rods that transmit the rotation from a motor placed outside the magnet.

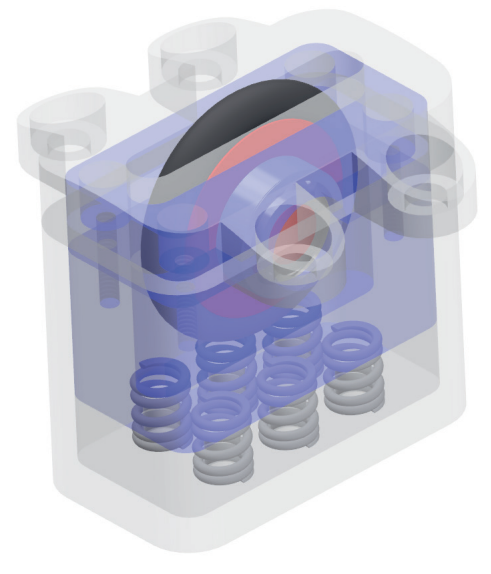

Fig. 2: Rendering of the stabilizing wheel.

The base mechanical structure of the probe was 
fabricated using 3D printing technology due to its complicated shape and because the prototype version was designed to accommodate multiple solutions for testing. The two main features to be tested are the positioning of the level meter, as well as supporting the probe in the magnet aperture. In the first tested variant, the level meter was mounted to the base of the scanner, which was held in position during the measurement using the stabilizing wheels as shown in Fig. 2.

The main goal of the wheels is to facilitate longitudinal displacements, while retaining the angular position of the base during the coil rotation.

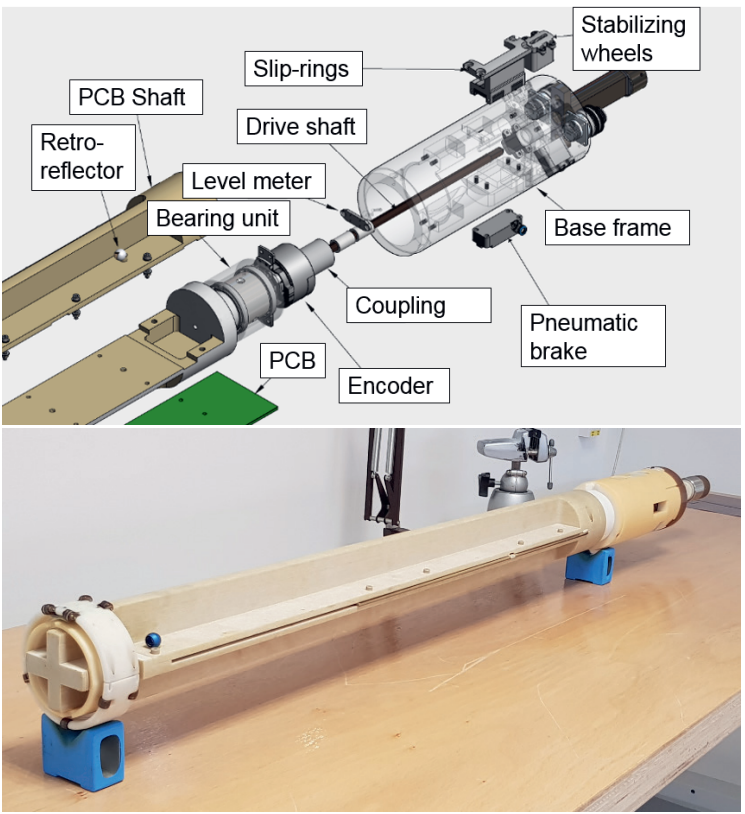

Fig. 3: First prototype measurement system. Top: exploded view of the probe base structure. Bottom: The picture of an assembled system.

Considering the high positioning accuracy required for the PCB coils, together with the size limitations for the $3 D$ printer, we decided that the support shaft for the PCB board should be machined from a glass fiber composite. The support shaft has been designed to allow mounting differently sized PCBs without major effort, in particular without disassembling the base structure. Moreover, the part is relatively easy to machine and modify thanks to parametric design. Therefore, it goes in line with the adaptability requirement; a single probe can be used for measuring magnets of different bore radii. The final design of the first prototype is shown in Fig. 3.

The fabricated PCBs (see Fig. 4) are equipped with two sets of five induction coils. To balance the cost and complexity of manufacturing we decided to use 24 active layers in the board. The number of turns per layer has been established by taking into account the clearances of $125 \mu \mathrm{m}$ between the tracks, and the copper track widths of $125 \mu \mathrm{m}$, to minimize the chances for short circuits or track breaks. Generally, we aimed at maximizing the number of turns, without reducing the average measurement radius of the coil below $35 \mathrm{~mm}$, which is derived from the measurement specification. Finally, we were able to fit 11 turns per layer, which resulted in a total of 264 coil-winding turns.

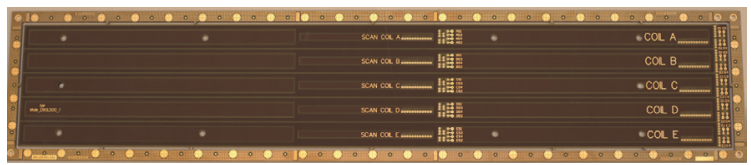

Fig. 4: The PCB board containing an array of 10 coils.

The $500 \mathrm{~mm}$-long coils have been designed to cover the entire length of the magnet end region and provide a high SNR. The smaller, nested coils, that span $100 \mathrm{~mm}$ can be used in case a higher longitudinal resolution is required. In the case of induction-coil arrays manufactured in PCB technology, adding the nested coils comes at a very little price both in design time and production cost.

To study the uncertainty in the measurements due to manufacturing tolerances in the PCB production and layer stacking, the "witness tracks" were inspected under a microscope; see Fig. 5.

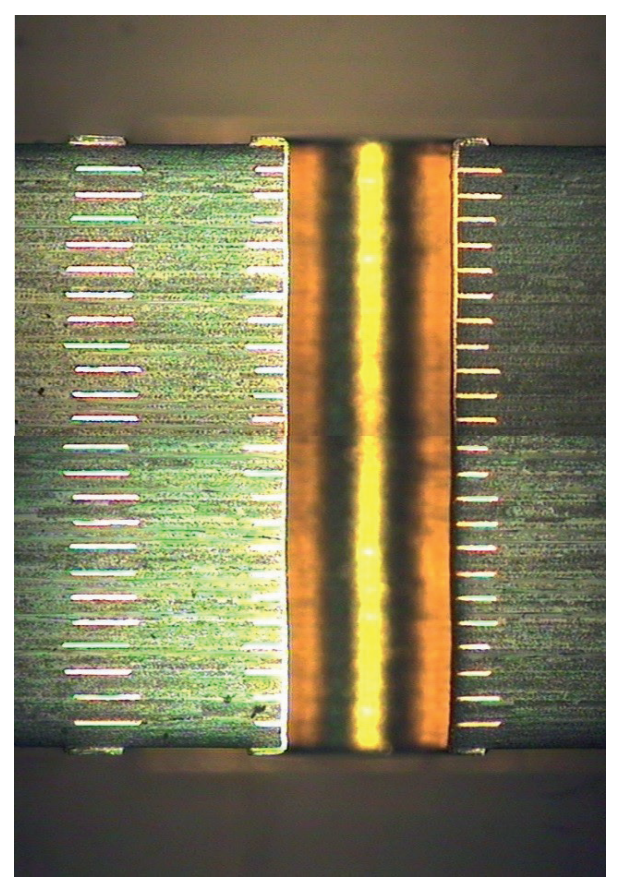

Fig. 5: The PCB stack cross-section under microscope.

By measuring the positions of the tracks, we established the accuracy of the layer positioning to be within $\pm 30 \mu \mathrm{m}$. Using the CERN field computation program ROXIE [9], we calculated the sensitivity factors of the coil with random errors 
of layer placement in this range of tolerance. The results of over 500 iterations show that for the coefficients of lower orders, the standard deviation in the sensitivity factors is negligible, while for the coefficients of higher orders (15-th and above) the standard deviation can be close to the percent range. This, in turn, would result in a relative error on the order of ppm in the measurement, as the higher order components in the field rarely exceed $100 \mathrm{ppm}$.

\section{Coil calibration}

The first step in the assembly process, was to measure the spanned surface of the PCB coils [4]. Even if one can rely on the optical measurement of the track position, the calibration step is necessary for verifying the correctness of the coil production and detecting the inter-turn shorts that cannot be detected by resistance measurements. The measured surface is used for computing the transfer function between the magnetic flux intercepted by the induction coil and the acquired voltage signal. The surface of the coil can be found by flipping it upside down in the uniform magnetic field of a reference magnet, while integrating the output voltage. The integration yields

$$
-\int_{0}^{t} V_{c} \mathrm{~d} t=\Phi-(-\Phi)=2 A \bar{B},
$$

where $\bar{B}$ is the average magnetic flux density, and $A$ is the surface spanned by the coil. The average surface measured for the three produced PCBs is $1.8729 \mathrm{~m}^{2}$. These results have been corrected for the resistance of the coil $(2.7 \mathrm{k} \Omega)$, as the input stage of the integrator has an impedance of $400 \mathrm{k} \Omega$. The measured values show very high homogeneity between the coils on one PCB as well as between the boards; the differences are in the order of $10^{-4} \mathrm{~m}^{2}$.

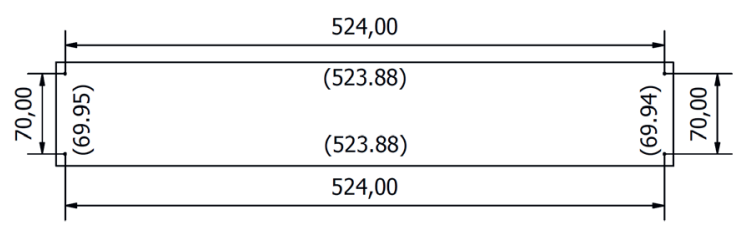

Fig. 6: Comparison of measured and design values for coil reference points. Measured values are in brackets.

We then compared the measurement results with the nominal surface from the PCB design and found a relative difference of approximately $0.1 \%$. The reason for this discrepancy is the shrinking of the PCB board during the production. A precise geometric measurement of the reference points on the board (see Fig. 6) provided values to calculate the appropriate shrinking factor, assuming homogeneous shrinking of the whole board. The computed area, corrected by the shrinking factor equals $1.8727 \mathrm{~m}^{2}$, which differs from the measured surfaces on the order of $10^{-4} \mathrm{~m}^{2}$. These values are very close to the standard deviation of the calibration results that is on the order of $10^{-5}$.

More PCB samples are needed to create proper statistics and identify the important factors for refining the design and fabrication process. The ultimate goal of these efforts is to be able to assume, with high certainty, that the final coil area corresponds to the design within the $10^{-5}$ relative tolerance. This would limit the role of the surface calibration to a mere electrical verification of the PCB coils. This is especially important for large PCBs that do not fit into the available reference magnets.

\section{Measurement results}

The first validation measurement campaign has been conducted in two reference quadrupoles used in the Magnetic Measurements (MM) section at CERN. Fig. 7 shows a measurement setup in one of the short magnets.

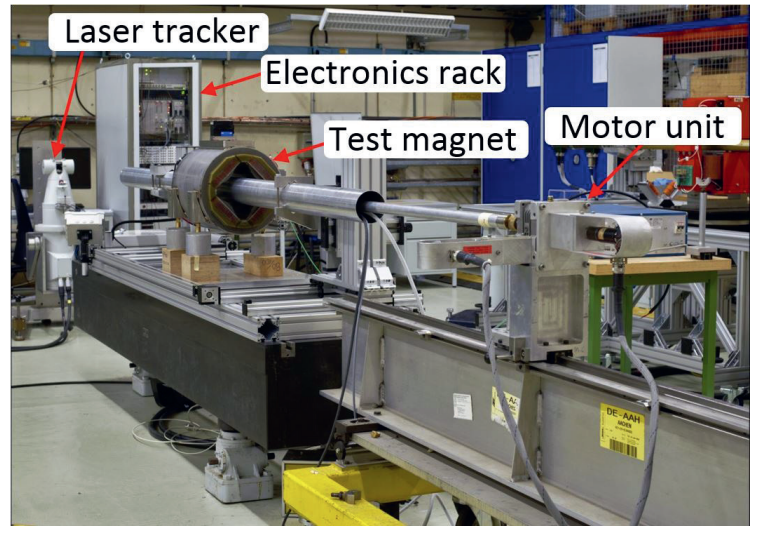

Fig. 7: Measurement setup with all system components. The probe is inside the tube that goes through the magnet.

Both magnets have been scanned along their entire length, using the laser tracker for longitudinal positioning as well as for tracking the rotation axis. In each position, the coils have been rotated 20 times in both directions to compensate for angular positioning errors and provide data for uncertainty analysis. The precision of local measurements shown in Table 3 and Fig. 8 is consistent among the different positions in both magnets.

Tab. 3: The precision of main field parameters measurement of a single position.

\begin{tabular}{|l|c|c|}
\hline Parameter & Unit & Repeatability \\
\hline Main field & [ppm] & 60 \\
Main field direction & [mrad] & 0.1 \\
Magnetic center & [mm] & 0.1 \\
\hline
\end{tabular}




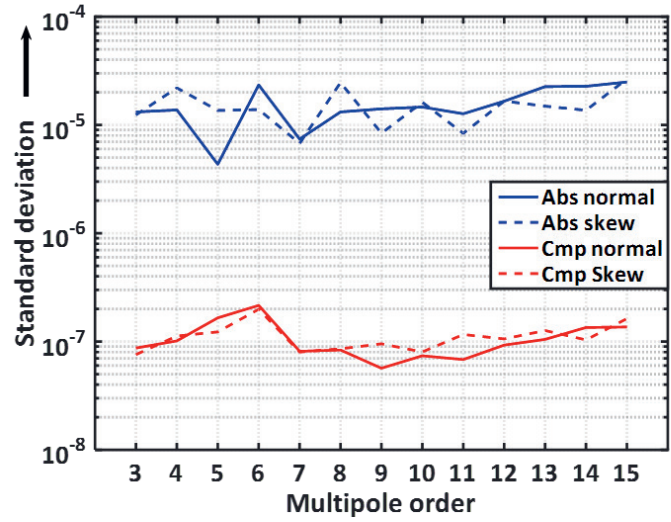

Fig. 8: The precision of field harmonics measurement of a single position.

The plot also shows the advantages of the compensation scheme for the the main field components. If the field harmonics are calculated using the signal from a single coil, the accuracy is at least 100 times worse. The quality of the coil production and positioning can be assessed by comparing the field strength measured by a single coil and the coils connected in a compensation scheme. The resulting proportional factor is commonly called the compensation (or bucking) ratio. As seen from the scale differences in Fig. 9 the achieved bucking ratio for dipole and quadrupole components are on the order of 1000.

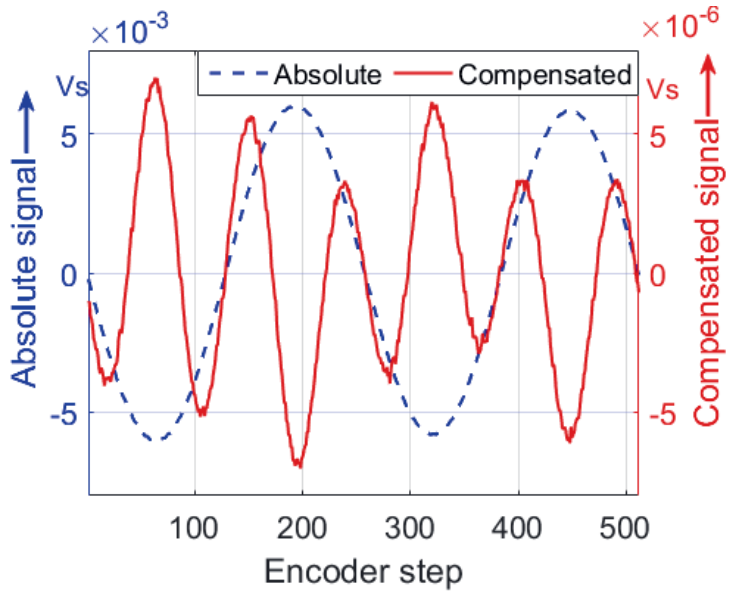

Fig. 9: The acquired integrated voltage between steps of the encoder over one rotation.

The integral values are computed from the local measurement results at all positions along the magnet. The local values have to be multiplied by the induction-coil length and then added together. In this case, two additional factors play an important role: the knowledge of the exact length of the coil and the precise longitudinal positioning, especially in the end regions of the magnet. The measurements have been conducted with both long and short coils several times to evaluate the repeatability of the system. The results are shown in Fig. 10 and Fig. 11.
Field strength at Rref $=\mathbf{3 5} \mathbf{m m}$

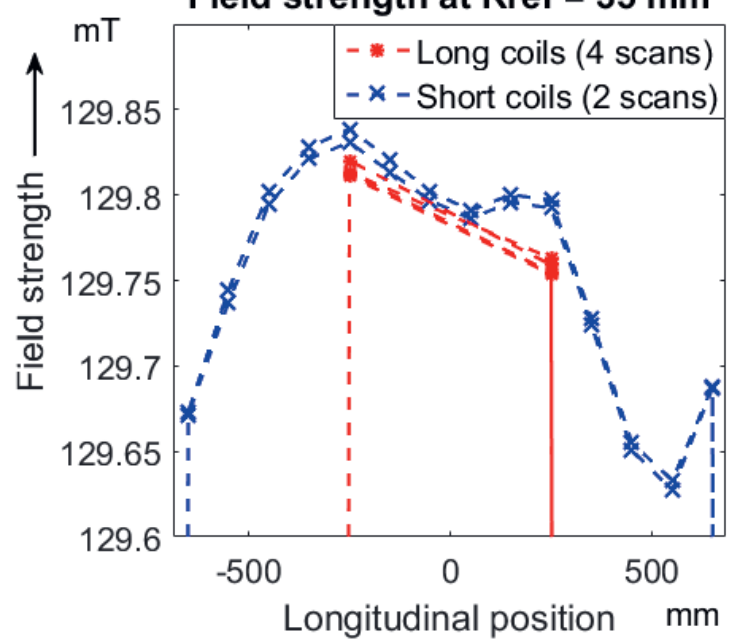

Fig. 10: Results of repeated scans with both long and short coils. External positions are removed from the plot to emphasize the details in the straight field region.

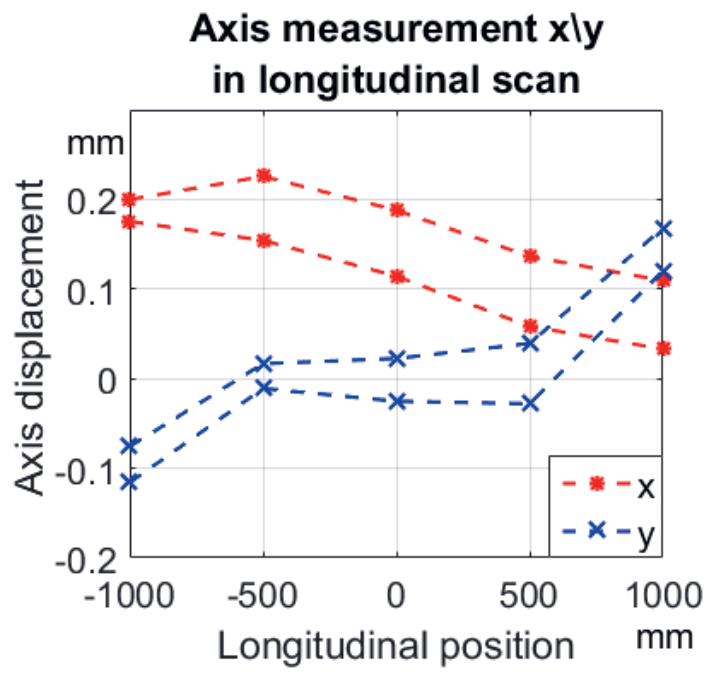

Fig. 11: Axis measurement results of two scans with long coils. The plot is centered at the average axis measured by a stretched wire system.

During the scan we discovered that the system mechanics is not precise enough, which results in movements of the base support during the rotation. Due to the compensation, the effect on the field strength and field harmonics measurements is negligible, but it has a noticeable influence on the axis and, especially, on the angle measurement. Since the integral values depend on the longitudinal positioning, the movement of the probe reduces the accuracy in their measurement as well.

The scan results have been compared to a stretched wire measurement that is the reference for integral values. The integrated gradient is in a good agreement with wire measurement; the 
accuracy is at the level of $300 \mathrm{ppm}$, which is a promising result. The axis is within $0.15 \mathrm{~mm}$ from the wire measurement. This difference between the results of two systems needs to be further investigated. Given the high repeatability of local measurements, it should still be possible to improve these values. In order to characterize the limits of the system performance, these results have been obtained in controlled and favorable environmental conditions. The reference quadrupole has a relatively high and pure field of approximately $130 \mathrm{mT}$ at the reference radius of $35 \mathrm{~mm}$.

After the first test campaign, we substituted the stabilizing wheels with a pneumatic brake and placed the level meter directly on the encoder. In this way, and by leveling the probe before the measurement, we can rely on the more linear range of the tilt sensor that moves with the encoder. This modification resulted in more stable and precise angle measurements, even without changing the mechanical structure. The accuracy of the field angle measurement has been established to be better than $0.1 \mathrm{mrad}$.

\section{Conclusions}

A new rotating coil magnetometer has been developed and characterized. The results of the measurements already indicate that its performance is at least as good as other existing systems, while offering the functionality of those systems combined. A prototype of a long insertionregion quadrupole for HL-LHC has been measured and the results have been used as feedback to the magnet development.

The adaptable design of the system and the use of $3 \mathrm{D}$ printing allowed us to easily test multiple configurations of the mechanical structure and induction coil setup. Some of the encountered mechanical issues have been addressed. While the measurement of the field strength and field harmonics is only slightly affected by mechanical imperfections (due to the compensation scheme), the measurements of the angle and magnetic axis require much higher mechanical precision. Ultimately, we aim to substitute the measurements with the stretched-wire system with the integrated measurements of the rotating coil scanner, substantially reducing the time and effort necessary for the measurements.

\section{Acknowledgements}

The authors would like to thank the Magnetic Measurements section laboratory and workshop staff for their help in assembling and testing the system. We would also like to thank D. Giloteaux, O. Dunkel and J. G. Perez for helpful suggestions and discussions. This work has been sponsored by the Wolfgang Gentner Programme of the Ger- man Federal Ministry of Education and Research (grant no. 05E15CHA)

\section{References}

[1] G. Apollinari et al. CERN Yellow Reports: Monographs, Vol 4 (2017): HighLuminosity Large Hadron Collider (HLLHC) Technical Design Report V. 0.1. 2017. DOI: $10.23731 /$ CYRM-2017-004.

[2] Pasquale Arpaia, Gianni Caiafa, and Stephan Russenschuck. "A Rotating-Coil Magnetometer for Scanning Transversal Field Harmonics in Accelerator Magnets". In: Scientific reports 9.1 (2019), p. 1491. DOI: $10.1038 / \mathrm{s} 41598-018-37371-3$.

[3] L. Bottura, M. Buzio, S. Pauletta, and N. Smirnov. "Measurement of magnetic axis in accelerator magnets: critical comparison of methods and instruments". In: Proceedings of the 23rd IEEE Instrumentation and Measurement Technology Conference, 2006, IMTC 2006. IEEE Operations Center, 2006, pp. 765-770. DOI: 10.1109/ IMTC . 2006. 328154.

[4] M. Buzio. "Fabrication and calibration of search coils". In: CAS - CERN Accelerator School: Specialised course on Magnets. Ed. by Daniel Brandt. CERN, 2009, pp. 387-421. DOI: 10 . 5170/CERN-2010004.

[5] W. G. Davies. "The theory of the measurement of magnetic multipole fields with rotating coil magnetometers". In: Nuclear Instruments and Methods in Physics Research Section A: Accelerators, Spectrometers, Detectors and Associated Equipment 311.3 (1992), pp. 399-436. DOI: 10. 1016/0168-9002 (92) 90637-J.

[6] J. DiMarco et al. "Application of PCB and FDM Technologies to Magnetic Measurement Probe System Development". In: IEEE Transactions on Applied Superconductivity 23.3 (2013), p. 9000505 . DOI: 10. 1109/TASC. 2012.2236596.

[7] J. DiMarco et al. "Field alignment of quadrupole magnets for the LHC interaction regions". In: IEEE Transactions on Appiled Superconductivity 10.1 (2000), pp. 127-130. DOI: 10.1109/77.828192.

[8] J. G. Perez, J. Billan, M. Buzio, P. Galbraith, D. GiloteauX, and V. Remondino. "Performance of the Room Temperature Systems for Magnetic Field Measurements of the LHC Superconducting Magnets". In: IEEE Transactions on Applied Superconductivity 16.2 (2006), pp. 269-272. DOI: 10.1109/TASC. 2006.871221 . 
[9] Stephan Russenschuck. Field computation for accelerator magnets: Analytical and numerical methods for electromagnetic design and optimization. Weinheim and Chichester, 2010. DOI: 10 . $1002 /$ 9783527635467.

[10] P. Schmüser. "Magnetic measurements of superconducting magnets and analysis of systematic errors". In: CAS - CERN Accelerator School. Ed. by Stuart Turner. CERN, 1992, pp. 240-273. DOI: 10.5170/CERN1992-005. 240. 